\title{
Class of exactly soluble models of one-dimensional spinless fermions and its application to the Tomonaga-Luttinger Hamiltonian with nonlinear dispersion
}

\author{
A.V. Rozhkov \\ Institute for Theoretical and Applied Electrodynamics RAS, \\ ul. Izhorskaya 13/19, 125412, Moscow, Russian Federation
}

(Dated: October 9, 2018)

\begin{abstract}
It is shown that for some special values of Hamiltonian parameters the Tomonaga-Luttinger model with nonlinear dispersion is unitary equivalent to the system of noninteracting fermions. For such parameter values the density-density propagator of the Tomonaga-Luttinger Hamiltonian with nonlinear dispersion can be found exactly. In a generic situation the exact solution can be used as a reference point around which a perturbative expansion in orders of certain irrelevant operators may be constructed.
\end{abstract}

\section{INTRODUCTION}

The Tomonaga-Luttinger model is the most important model of one-dimensional interacting electron physics. The model describes two Fermi points of right-moving $(\mathrm{R})$ and left-moving $(\mathrm{L})$ electrons. The electrons interact through a two-body short-range potential:

$$
\begin{array}{r}
H_{\mathrm{TL}}=H_{\mathrm{kin}}+H_{\mathrm{int}}, \\
H_{\mathrm{kin}}=i v_{\mathrm{F}} \int_{-L / 2}^{L / 2} d x\left(: \psi_{\mathrm{L}}^{\dagger} \nabla \psi_{\mathrm{L}}:-: \psi_{\mathrm{R}}^{\dagger} \nabla \psi_{\mathrm{R}}:\right), \\
H_{\mathrm{int}}=\int d x d x^{\prime} \hat{g}\left(x-x^{\prime}\right) \rho_{\mathrm{L}}(x) \rho_{\mathrm{R}}\left(x^{\prime}\right), \\
\rho_{\mathrm{L}, \mathrm{R}}=: \psi_{\mathrm{L}, \mathrm{R}}^{\dagger} \psi_{\mathrm{L}, \mathrm{R}}:,
\end{array}
$$

where function $\hat{g}(x)$ vanishes for $\Lambda|x| \gg 1$. The parameter $\Lambda$ plays a role of the ultraviolet cutoff. The symbol :... : stands for the normal ordering with respect to the noninteracting ground state.

The kinetic energy (2) of the model (1) is a linear function of the electron momentum. It is a necessary condition for the exact solubility of $H_{\mathrm{TL}}$ : when the kinetic energy is linear one can apply bosonization to find the excitation spectrum and the correlation functions of the model $[1,2]$.

However, there are situations in which we must extend the model by including the nonlinear dispersion term:

$$
H_{\mathrm{nl}}=v_{\mathrm{F}}^{\prime} \int d x\left(: \nabla \psi_{\mathrm{L}}^{\dagger} \nabla \psi_{\mathrm{L}}:+: \nabla \psi_{\mathrm{R}}^{\dagger} \nabla \psi_{\mathrm{R}}:\right) .
$$

Such generalization immediately introduces serious complications: the bosons become interacting.

Another extension of the system (1) where the bosonization fails is the quasi-one-dimensional conductor: an array of one-dimensional Tomonaga-Luttinger wires coupled by transverse hopping and interaction. Bosonization works poorly for the transverse hopping has no simple expression in terms of the Tomonaga-Luttinger bosons.
Thus, some researchers began developing 'fermionic' approaches to the model. In a variety of contexts the one-dimensional electron systems were described in terms of the composite fermionic degrees of freedom $[3,4,5]$. More to the topic of the present paper are Ref. $[6,7,8]$ which we briefly discuss below.

In Ref. $[6,7]$ the authors studied the effect of nonlinear dispersion $v_{\mathrm{F}}^{\prime}$ on the density-density propagator of the Tomonaga-Luttinger Hamiltonian. They avoided using the bosonization and instead worked with the bare fermionic degrees of freedom.

The quasi-one-dimensional case was investigated in Ref. [8] where the hybrid boson-fermion representation for the quasi-one-dimensional Hamiltonian was proposed. The latter representation describes the highenergy one-dimensional degrees of freedom in terms of the Tomonaga-Luttinger bosons while the low-energy threedimensional degrees of freedom as fermionic quasiparticles.

Despite their success, both approaches are not systematic. Rather, they were custom-made for particular tasks at hand. The systematic method to handle the generalized problem (1)-(5) was proposed by the author in Ref. [9]. The main idea of the method is to apply a certain canonical transformation to (1)-(5) which kills marginal (in the renormalization group sense) interaction $H_{\text {int }}$. Such transformation maps the original model on the model of fermionic quasiparticles weakly interacting through an irrelevant operator. Due to the irrelevance of the quasiparticle interaction the usual perturbation theory is applicable and the quasiparticles near the Fermi points are free.

This approach allows one to calculate the retarded density-density correlation function of the TomonagaLuttinger model with nonlinear dispersion to the zeroth order in the quasiparticle interaction [9].

The developments described above solve the longstanding problem of the Tomonaga-Luttinger model with the nonlinear dispersion. The purpose of this paper is refinements of the method: we will show that if, in addition to the nonlinear dispersion, one supplies the TomonagaLuttinger Hamiltonian with a certain type of irrelevant interactions the Hamiltonian becomes exactly soluble. The system at and near this solubility point is studied 
in this paper.

We proceed by noting that the classical formulation of the Tomonaga-Luttinger model with nonlinear dispersion (1)-(5) is somewhat inconsistent. It is trivial to check that the scaling dimension of $H_{\mathrm{nl}}$ is equal to 3 . Indeed, the field operator has dimension of $1 / 2$, the dimension of $\nabla$ is unity. Therefore, the dimension of the whole operator is 3 . In addition to $H_{\mathrm{nl}}$, there is one and only one operator whose scaling dimension is also 3 :

$$
\begin{aligned}
& H_{\mathrm{int}}^{\prime}=i g^{\prime} \int d x\left[\rho_{\mathrm{R}}\left(: \psi_{\mathrm{L}}^{\dagger}\left(\nabla \psi_{\mathrm{L}}\right):-:\left(\nabla \psi_{\mathrm{L}}^{\dagger}\right) \psi_{\mathrm{L}}:\right)\right. \\
&\left.-\rho_{\mathrm{L}}\left(: \psi_{\mathrm{R}}^{\dagger}\left(\nabla \psi_{\mathrm{R}}\right):-:\left(\nabla \psi_{\mathrm{R}}^{\dagger}\right) \psi_{\mathrm{R}}:\right)\right]
\end{aligned}
$$

From the renormalization group view point if we are interested in the effect of $H_{\mathrm{nl}}$ we must include $H_{\mathrm{int}}^{\prime}$ as well.

Initially, it looks as if such modification leads to substantial additional difficulties. Yet, despite this expectations, we will see that the operator $H_{\mathrm{int}}^{\prime}$ does not make our problem more complicated at all. Moreover, if $g^{\prime}, v_{\mathrm{F}}^{\prime}$, $g$ and $v_{\mathrm{F}}$ satisfy some special relation the Hamiltonian

$$
\begin{aligned}
H_{\mathrm{ex}} & =H_{\mathrm{TL}}+H_{\mathrm{nl}}+H_{\mathrm{int}}^{\prime} \\
& +(\text { irrelevant operators with scaling } \operatorname{dim} \geq 4)
\end{aligned}
$$

is equivalent to the system of noninteracting fermions. Thus, the system becomes exactly soluble.

The exact solubility gives us a chance to access qualities otherwise hidden from the analytical investigation. For example, the spectrum of the Hamiltonian and the statistics of excitations can be determined. Additionally, the density-density propagator can be calculated as well. It is proportional to the propagator of the free fermions.

Of course, a generic model (which we denote as $H_{\mathrm{G}}$, where ' $G$ ' stands for 'generic') deviates from the exactly soluble case. However, it can be shown that if such deviations are weak we can study the generic model with the help of perturbation theory in orders of $\left(H_{\mathrm{G}}-H_{\mathrm{ex}}\right)$. In particular, the propagator of the exactly soluble Hamiltonian is the zeroth order approximation to the propagator of $H_{\mathrm{G}}$.

The paper is organized as follows. The exactly soluble model is derived in Sect.II. Since the latter Section is very technical the reader might get lost in the details. For those who are not specifically interested in all the minutia there is Sect.III where we give concise nontechnical overview of the presented derivations. This Section is fairly self-contained so that the reader ready to settle for just a heuristic argumentation may skip Sect.II. The situation of weak deviation from the point of exact solubility is discussed in Sect.IV. Sect.V is dedicated to the application of Sect.IV ideas to calculations of observables. In Sect.VI we compare our approach with the results available in the literature. Technically involved calculations are relegated to Appendix.

\section{EXACTLY SOLUBLE MODEL}

We start with the quadratic Hamiltonian:

$$
\begin{array}{r}
H_{0}=H_{\mathrm{kin}}+H_{\mathrm{nl}}+\ldots= \\
\sum_{p} \int d x\left\{i p \tilde{v}_{\mathrm{F}}: \psi_{p}^{\dagger}\left(\nabla \psi_{p}\right):+\tilde{v}_{\mathrm{F}}^{\prime}:\left(\nabla \psi_{p}^{\dagger}\right)\left(\nabla \psi_{p}\right):\right\}+\ldots
\end{array}
$$

where ellipsis stand for quadratic terms which contain more space derivatives. We must assume their presence to avoid creating spurious Fermi points besides those located at $q=0$. From the practical point these terms cause us no trouble. They will be discussed in Sect.IV, together with other terms whose scaling dimension is 4 and higher. The summation in eq.(8) runs over the chirality index $p$ which is equal 1 for left-movers and -1 for right-movers.

As we have mentioned above the colons denote the normal ordering with respect to the noninteracting ground state $|0\rangle$. Specifically, the normal order is defined by the equations:

$$
\begin{array}{r}
\psi_{p}^{\dagger}(x) \psi_{p}(y)=: \psi_{p}^{\dagger}(x) \psi_{p}(y):+s_{p}(x-y),(9) \\
\psi_{p}^{\dagger}(x) \psi_{p}(y) \psi_{p}^{\dagger}\left(x^{\prime}\right) \psi_{p}\left(y^{\prime}\right)=: \psi_{p}^{\dagger}(x) \psi_{p}(y) \psi_{p}^{\dagger}\left(x^{\prime}\right) \psi_{p}\left(y^{\prime}\right):(10) \\
+s_{p}(x-y): \psi_{p}^{\dagger}\left(x^{\prime}\right) \psi_{p}\left(y^{\prime}\right):+s_{p}\left(x^{\prime}-y^{\prime}\right): \psi_{p}^{\dagger}(x) \psi_{p}(y): \\
+s_{p}\left(x-y^{\prime}\right): \psi_{p}(y) \psi_{p}^{\dagger}\left(x^{\prime}\right):+s_{p}\left(y-x^{\prime}\right): \psi_{p}^{\dagger}(x) \psi_{p}\left(y^{\prime}\right): \\
+s_{p}(x-y) s_{p}\left(x^{\prime}-y^{\prime}\right)+s_{p}\left(x-y^{\prime}\right) s_{p}\left(y-x^{\prime}\right), \\
s_{p}(x)=\left\langle 0\left|\psi_{p}^{\dagger}(x) \psi_{p}(0)\right| 0\right\rangle=(11) \\
\left\langle 0\left|\psi_{p}(x) \psi_{p}^{\dagger}(0)\right| 0\right\rangle=\frac{p}{2 \pi i(x-i p 0)} .
\end{array}
$$

Obviously, the Hamiltonian (8) is trivially soluble. The spectrum of the fermionic excitations is

$$
\tilde{\varepsilon}_{p q}=p \tilde{v}_{\mathrm{F}} q+\tilde{v}_{\mathrm{F}}^{\prime} q^{2}+O\left(q^{3}\right) .
$$

The contribution $O\left(q^{3}\right)$ comes from the terms of eq. (8) denoted by the ellipsis. These terms guarantee that $\tilde{\varepsilon}_{p q}=$ 0 for $q=0$ only.

The density-density propagator for the model (8)

$$
\mathcal{D}_{q \omega}^{0}=\frac{1}{4 \pi \tilde{v}_{\mathrm{F}}^{\prime} q} \log \left\{\frac{\omega^{2}+\left(\tilde{v}_{\mathrm{F}} q-\tilde{v}_{\mathrm{F}}^{\prime} q^{2}\right)^{2}}{\omega^{2}+\left(\tilde{v}_{\mathrm{F}} q+\tilde{v}_{\mathrm{F}}^{\prime} q^{2}\right)^{2}}\right\}
$$

can be calculated without much effort.

Now we define the unitary transformation $U$ :

$$
\begin{array}{r}
U=\mathrm{e}^{\Omega}, \\
\Omega=-\frac{1}{2} \sum_{q \neq 0} \sum_{p} p \frac{\alpha_{q}}{n_{q}} \rho_{p-q} \rho_{-p q}, \\
n_{q}=\frac{L q}{2 \pi}, \quad \rho_{p q}=\int d x \mathrm{e}^{-i q x} \rho_{p}(x),
\end{array}
$$

and transform with its help the operator $H_{0}$ :

$$
H_{\mathrm{ex}}=U H_{0} U^{\dagger} \text {. }
$$


The subscript 'ex' stands for 'exact' since the Hamiltonian $H_{\text {ex }}$ is unitary equivalent to the quadratic Hamiltonian (8) which implies exact solubility of $H_{\mathrm{ex}}$.

The coefficients $\alpha$ 's from eq.(15) uniquely specify the operator $U$. We impose on them the following conditions:

$$
\begin{array}{r}
\alpha_{q}=\alpha_{-q}, \\
\left.\alpha_{q} \approx \alpha_{q}\right|_{q=0} \text { for }|q|<\Lambda, \\
\alpha_{q} \rightarrow 0 \text { for }|q| \gg \Lambda .
\end{array}
$$

These conditions mean that $U$ when acting in the Fock space of Hamiltonian (8) cannot create highly excited single-fermion states. Loosely speaking, the action of $U$ is confined to the region $|q|<\Lambda$ near the Fermi points. This guarantees that the interactions of $H_{\mathrm{ex}}$ have proper ultraviolet cutoff of $\Lambda$.

In order to find $H_{\text {ex }}$ explicitly we need the following commutation rules:

$$
\begin{array}{r}
{\left[\rho_{p q}, \rho_{p^{\prime}-q^{\prime}}\right]=\delta_{p p^{\prime}} \delta_{q q^{\prime}} p n_{q},} \\
{\left[\rho_{p q}, \psi_{p^{\prime}}^{\dagger}(x)\right]=\delta_{p p^{\prime}} \mathrm{e}^{-i q x} \psi_{p^{\prime}}^{\dagger}(x),}
\end{array}
$$

which are proven in $[1,10]$.

Next, let us study how operator $U$ acts on different observables. With the help of eq.(21) it is possible to show that for $q \neq 0$ :

$$
\begin{array}{r}
U \rho_{p q} U^{\dagger}=u\left(\alpha_{q}\right) \rho_{p q}+v\left(\alpha_{q}\right) \rho_{-p q}, \\
u\left(\alpha_{q}\right)=u_{q}=\operatorname{ch} \alpha_{q}, v\left(\alpha_{q}\right)=v_{q}=\operatorname{sh} \alpha_{q}, \\
u_{q}^{2}-v_{q}^{2}=1 .
\end{array}
$$

For zero modes we have:

$$
\begin{gathered}
U N_{p} U^{\dagger}=N_{p}, \\
N_{p}=\left.\rho_{p q}\right|_{q=0} .
\end{gathered}
$$

Such transformation rule is a consequence of definition (15), in which $\Omega$ commutes with $N_{p}$.

It is convenient to define the following symbols:

$$
\begin{array}{r}
\rho_{p}^{u}(x)=\int d y \hat{u}(y) \rho_{p}(x-y)=\left(\hat{u} * \rho_{p}\right)(x), \\
\rho_{p}^{v}(x)=\int d y \hat{v}(y) \rho_{p}(x-y)=\left(\hat{v} * \rho_{p}\right)(x), \\
\hat{u}(x)=L^{-1} \sum_{q} u_{q} \mathrm{e}^{i q x}=\int \frac{d q}{2 \pi} u_{q} \mathrm{e}^{i q x}, \\
\hat{v}(x)=L^{-1} \sum_{q} v_{q} \mathrm{e}^{i q x}=\int \frac{d q}{2 \pi} v_{q} \mathrm{e}^{i q x} .
\end{array}
$$

Here the asterisks stand for convolutions of two functions.

Due to eq.(20) we have $u_{q}=1$ and $v_{q}=0$ for $|q| \gg \Lambda$. Therefore, $\hat{v}(x)$ is well defined, whereas $\hat{u}(x)$ is singular:

$$
\begin{aligned}
\hat{u}(x) & =\delta(x)+\hat{w}(x), \\
\hat{w}(x)=L^{-1} \sum_{q} w_{q} \mathrm{e}^{i q x} & =\int \frac{d q}{2 \pi} w_{q} \mathrm{e}^{i q x}, \\
w_{q}=u_{q}-1 & \rightarrow 0 \text { for }|q| \gg \Lambda,
\end{aligned}
$$

where $\hat{w}(x)$ is well-defined.

Both $\hat{v}(x)$ and $\hat{w}(x)$ are even functions of $x$. They vary slowly on the scale of $(1 / \Lambda)$ and vanish for $|x| \gg(1 / \Lambda)$.

We express the action of $U$ on $\rho_{p}(x)$ in the following manner:

$$
\begin{array}{r}
U \rho_{p}(x) U^{\dagger}=\rho_{p}^{u}(x)+\rho_{-p}^{v}(x)+\delta \rho_{p 0} \\
=\rho_{p}(x)+\rho_{p}^{w}(x)+\rho_{-p}^{v}(x)+\delta \rho_{p 0}, \\
\rho_{p}^{w}=\hat{w} * \rho_{p}, \\
\delta \rho_{p 0}=\frac{1}{L}\left[\left(1-u_{0}\right) N_{p}-v_{0} N_{-p}\right] \\
=-\frac{1}{L}\left[w_{0} N_{p}+v_{0} N_{-p}\right], \\
u_{0}=\left.u_{q}\right|_{q=0}, v_{0}=\left.v_{q}\right|_{q=0}, w_{0}=\left.w_{q}\right|_{q=0} .
\end{array}
$$

The obtained result for $U \rho U^{\dagger}$ can be used to find $U H_{\text {kin }} U^{\dagger}$. We prove in Appendix that:

$$
\begin{array}{r}
\frac{i p}{2}\left[: \psi_{p}^{\dagger}(x)\left(\nabla \psi_{p}(x)\right):-:\left(\nabla \psi_{p}^{\dagger}(x)\right) \psi_{p}(x):\right] \\
=\pi \lim _{y \rightarrow x}\left\{\rho_{p}(x) \rho_{p}(y)-b_{p}(x-y)\right\}, \\
b_{p}(x)=\left\langle 0\left|\rho_{p}(x) \rho_{p}(0)\right| 0\right\rangle=s_{p}^{2}(x) .
\end{array}
$$

These formulas express the kinetic energy density in terms of the density operators. Since we know how the latter are transformed under the action of $U$ we determine $U H_{\text {kin }} U^{\dagger}$ easily, as the derivation below demonstrates.

First, introduce the notation:

$$
\begin{array}{r}
: \rho_{p}(x) \rho_{p}(y):=\rho_{p}(x) \rho_{p}(y)-b_{p}(x-y), \\
: \rho_{p}(x) \rho_{p}(y) \rho_{p}(z):=\rho_{p}(x) \rho_{p}(y) \rho_{p}(z)- \\
b_{p}(x-y) \rho_{p}(z)-b_{p}(x-z) \rho_{p}(y)-b_{p}(y-z) \rho_{p}(x) .
\end{array}
$$

In the bosonization framework the above equations define the normal ordering of the bosonic operators. In this paper we do not introduce the Tomonaga-Luttinger bosons. Thus, the symbols $: \rho \rho$ : and $: \rho \rho \rho$ : must be understood as a shorthand notation for expressions like eq.(41) and eq.(42) without further hidden field-theoretical meaning. The reason for using normal ordered products is that such objects are well defined analytically at any values of $x, y$ and $z$ while unordered products have singularities when arguments coincide (e.g, the unordered product $\rho_{p}(x) \rho_{p}(y)$ has a second order pole at $x=y$ ).

The new notation allows us to reformulate eq.(39) in a more compact way:

$$
\frac{i p}{2}\left[: \psi_{p}^{\dagger}\left(\nabla \psi_{p}\right):-:\left(\nabla \psi_{p}^{\dagger}\right) \psi_{p}:\right]=\pi: \rho_{p}^{2}: .
$$

In the bosonization context this equality corresponds to the bosonization of the kinetic energy $[1,10]$.

Using eq.(39) and eq.(43) we find:

$$
\begin{array}{r}
U H_{\mathrm{kin}} U^{\dagger}=\pi \tilde{v}_{\mathrm{F}} \sum_{p} \int d x U: \rho_{p}^{2}: U^{\dagger}= \\
\pi \tilde{v}_{\mathrm{F}} \int d x \sum_{p} \lim _{y \rightarrow x} U\left\{\rho_{p}(x) \rho_{p}(y)-b_{p}(x-y)\right\} U^{\dagger} .
\end{array}
$$


The expression under the limit sign is equal to:

$$
\begin{array}{r}
U\left\{\rho_{p}(x) \rho_{p}(y)-b_{p}(x-y)\right\} U^{\dagger}= \\
\left(\rho_{p}^{u}(x)+\rho_{-p}^{v}(x)+\delta \rho_{p 0}\right)\left(\rho_{p}^{u}(y)+\rho_{-p}^{v}(y)+\delta \rho_{p 0}\right) \\
-b_{p}(x-y)
\end{array}
$$

In this equation we need to normal order $\rho$ operators according to definition eq.(41):

$$
\rho_{p}^{u}(x) \rho_{p}^{u}(y)=: \rho_{p}^{u}(x) \rho_{p}^{u}(y):+\left(\hat{u} * b_{p} * \hat{u}\right)(x-y) .
$$

It is easy to show with the help of eq.(25) that for any function $f(x)$

$$
(\hat{u} * f * \hat{u})(x)=f(x)+(\hat{v} * f * \hat{v})(x) .
$$

Therefore, eq.(46) may be written differently:

$$
\begin{gathered}
\rho_{p}^{u}(x) \rho_{p}^{u}(y)=: \rho_{p}^{u}(x) \rho_{p}^{u}(y):+ \\
b_{p}(x-y)+\left(\hat{v} * b_{p} * \hat{v}\right)(x-y) .
\end{gathered}
$$

Finally:

$$
\rho_{-p}^{v}(x) \rho_{-p}^{v}(y)=: \rho_{-p}^{v}(x) \rho_{-p}^{v}(y):+\left(\hat{v} * b_{-p} * \hat{v}\right)(x-y)
$$

Collecting all terms together one finds:

$$
\begin{array}{r}
U H_{\mathrm{kin}} U^{\dagger}=\pi \tilde{v}_{\mathrm{F}} \int d x \sum_{p}:\left(\rho_{p}^{u}+\rho_{-p}^{v}+\delta \rho_{p 0}\right)^{2}: \\
+2 \pi c \tilde{v}_{\mathrm{F}} \Lambda^{2} L,
\end{array}
$$

where $c$ is the dimensionless non-universal $c$-number:

$$
c=\Lambda^{-2}\left(\hat{v} *\left(b_{\mathrm{L}}+b_{\mathrm{R}}\right) * \hat{v}\right)(0)=\int \frac{d q}{(2 \pi \Lambda)^{2}}|q| v_{q}^{2} .
$$

In eq.(50) contributions proportional to $\delta \rho_{p 0}$ or $\left(\delta \rho_{p 0}\right)^{2}$ are $O\left(L^{-1}\right)$. Consequently, they may be neglected. The additive constant (the term proportional to $c$ ) can be neglected as well. With this in mind we determine:

$$
\pi \tilde{v}_{\mathrm{F}} \int d x\left[\sum_{p}\left\{:\left(\rho_{p}^{u}\right)^{2}:+:\left(\rho_{p}^{v}\right)^{2}:\right\}+2 \rho_{\mathrm{L}}^{u} \rho_{\mathrm{R}}^{v}+2 \rho_{\mathrm{L} i n}^{v} U_{\mathrm{R}}^{u}\right] .
$$

The expression in the curly brackets has to be transformed:

$$
\int d x\left\{:\left(\rho_{p}^{u}\right)^{2}:+:\left(\rho_{p}^{v}\right)^{2}:\right\}=\int d x\left\{: \rho_{p}^{2}:+2:\left(\rho_{p}^{v}\right)^{2}:\right\} .
$$

The second term can be written as:

$$
\begin{array}{r}
2 \int d x:\left(\rho_{p}^{v}\right)^{2}:= \\
2 \int d x d x^{\prime} d x^{\prime \prime} \hat{v}\left(x-x^{\prime}\right) \hat{v}\left(x-x^{\prime \prime}\right): \rho_{p}\left(x^{\prime}\right) \rho_{p}\left(x^{\prime \prime}\right):= \\
2 \int d x^{\prime} d x^{\prime \prime}(\hat{v} * \hat{v})\left(x^{\prime}-x^{\prime \prime}\right): \rho_{p}\left(x^{\prime}\right) \rho_{p}\left(x^{\prime \prime}\right):= \\
2 c_{0}^{v} \int d x: \rho_{p}^{2}:+h_{\mathrm{int}}^{(4)}
\end{array}
$$

The term $h_{\text {int }}^{(4)}$ is a sum of irrelevant operators whose scaling dimensions are 4 and higher. That is why we put such superscript at $h$ :

$$
\begin{array}{r}
h_{\mathrm{int}}^{(4)}=2 \sum_{n=1}^{\infty} c_{n}^{v} \Lambda^{-2 n} \int d x: \rho_{p}\left(\nabla^{2 n} \rho_{p}\right):, \\
c_{n}^{v}=\frac{\Lambda^{2 n}}{(2 n) !} \int d x x^{2 n}(\hat{v} * \hat{v})(x), \\
c_{0}^{v}=\int d x(\hat{v} * \hat{v})(x)=v_{0}^{2} .
\end{array}
$$

We establish:

$$
\begin{gathered}
\int d x\left\{:\left(\rho_{p}^{u}\right)^{2}:+:\left(\rho_{p}^{v}\right)^{2}:\right\}=\int d x\left(1+2 c_{0}^{v}\right): \rho_{p}^{2}:+h_{\text {int }}^{(4)}(58) \\
=\left(1+2 c_{0}^{v}\right) \frac{i p}{\pi} \int d x: \psi_{p}^{\dagger} \nabla \psi_{p}:+h_{\text {int }}^{(4)} .
\end{gathered}
$$

Here we used eq.(43) to transform $: \rho_{p}^{2}$ :

The $: \rho_{\mathrm{L}} \rho_{\mathrm{R}}$ : term of eq.(52) can be written as:

$$
\begin{array}{r}
2 \pi \tilde{v}_{\mathrm{F}} \int d x\left(\rho_{\mathrm{L}}^{u} \rho_{\mathrm{R}}^{v}+\rho_{\mathrm{L}}^{v} \rho_{\mathrm{R}}^{u}\right)= \\
\int d x d x^{\prime} \hat{g}\left(x-x^{\prime}\right) \rho_{\mathrm{L}}(x) \rho_{\mathrm{R}}\left(x^{\prime}\right), \\
\hat{g}(x)=4 \pi \tilde{v}_{\mathrm{F}}(v * u)(x) \Leftrightarrow g_{q}=4 \pi \tilde{v}_{\mathrm{F}} v_{q} u_{q} .
\end{array}
$$

Ultimately, we obtain the following expression for $U H_{\mathrm{kin}} U^{\dagger}$ :

$$
\begin{array}{r}
U H_{\mathrm{kin}} U^{\dagger}=i v_{\mathrm{F}} \int_{-L / 2}^{L / 2} d x\left(: \psi_{\mathrm{L}}^{\dagger} \nabla \psi_{\mathrm{L}}:-: \psi_{\mathrm{R}}^{\dagger} \nabla \psi_{\mathrm{R}}:\right) \\
+\int d x d x^{\prime} \hat{g}\left(x-x^{\prime}\right) \rho_{\mathrm{L}}(x) \rho_{\mathrm{R}}\left(x^{\prime}\right)+\pi \tilde{v}_{\mathrm{F}} h_{\mathrm{int}}^{(4)} \\
=H_{\mathrm{TL}}+\pi \tilde{v}_{\mathrm{F}} h_{\mathrm{int}}^{(4)} \\
v_{\mathrm{F}}=\tilde{v}_{\mathrm{F}}\left(1+2 c_{0}^{v}\right)=\tilde{v}_{\mathrm{F}}\left(1+2 v_{0}^{2}\right) .
\end{array}
$$

It is clear from eq.(61) that up to the linear combination of irrelevant operators $h_{\text {int }}^{(4)}$ the classical TomonagaLuttinger model is unitary equivalent to the model of free fermions with the Hamiltonian $H_{\text {kin }}$. This was shown in [9].

At first sight it appears that our ability to map the Tomonaga-Luttinger Hamiltonian on the free fermionic quasiparticles is incompatible with the known fact that there is no quasiparticle pole in the Tomonaga-Luttinger single-electron propagator. The resolution of this paradox is simple [9]: the overlap of the physical electron state and the quasiparticle state is zero. Thus, the singlequasiparticle propagator

$$
\begin{array}{r}
\mathcal{G}_{\mathrm{qp}}(x, \tau)=-\left\langle 0\left|\mathcal{T}_{\tau}\left\{\psi_{p}(x, \tau) \psi_{p}^{\dagger}(0,0)\right\}\right| 0\right\rangle, \\
\psi_{p}(x, \tau)=\mathrm{e}^{\tau H_{\mathrm{kin}}} \psi_{p}(x) \mathrm{e}^{-\tau H_{\mathrm{kin}}},
\end{array}
$$

does have a pole but such an object has no physical significance. Because of the orthogonality catastrophe the 
physically important single-electron propagator

$$
\begin{array}{r}
\mathcal{G}_{\mathrm{TL}}(x, \tau)=-\left\langle 0_{\mathrm{TL}}\left|\mathcal{T}_{\tau}\left\{\psi_{p}(x, \tau) \psi_{p}^{\dagger}(0,0)\right\}\right| 0_{\mathrm{TL}}\right\rangle, \\
\psi_{p}(x, \tau)=\mathrm{e}^{\tau H_{\mathrm{TL}}} \psi_{p}(x) \mathrm{e}^{-\tau H_{\mathrm{TL}}}, \\
\left|0_{\mathrm{TL}}\right\rangle=U|0\rangle-H_{\mathrm{TL}} \text { ground state }
\end{array}
$$

is not connected to the quasiparticle propagator in a Fermi liquid manner: $\mathcal{G}_{\mathrm{TL}}(q, \omega) \neq Z \mathcal{G}_{\mathrm{qp}}(q, \omega)+\mathcal{G}_{\text {reg }}(q, \omega)$. The Tomonaga-Luttinger single-electron propagator was derived with the help of representation (61) in Ref.[9].

It is interesting to establish the relation between the Tomonaga-Luttinger Hamiltonian parameters $v_{\mathrm{F}}, g$ and the free fermion parameter $\tilde{v}_{\mathrm{F}}$. If we define $g_{0}=\left.g_{q}\right|_{q=0}=$ $4 \pi \tilde{v}_{\mathrm{F}} u_{0} v_{0}$ we can write:

$$
\begin{array}{r}
\left(\frac{g_{0}}{2 \pi}\right)^{2}=4 \tilde{v}_{\mathrm{F}}^{2} v_{0}^{2}\left(1+v_{0}^{2}\right)=v_{\mathrm{F}}^{2}-\tilde{v}_{\mathrm{F}}^{2} \Rightarrow \\
\tilde{v}_{\mathrm{F}}=v_{\mathrm{F}} \sqrt{1-\left(\frac{g_{0}}{2 \pi v_{\mathrm{F}}}\right)^{2}} .
\end{array}
$$

The latter formula is very well known in the bosonization theory. It gives the dependence of the plasmon velocity on the bare Fermi velocity $v_{\mathrm{F}}$ and the interaction constant $g_{0}$. The latter interpretation does not contradict to eq. (69) for the plasmon mode in the fermion system with the Hamiltonian $H_{\text {kin }}$ propagates with the velocity $\tilde{v}_{\mathrm{F}}$.

Our next task is to find $U H_{\mathrm{nl}} U^{\dagger}$. We prove in Appendix that:

$$
:\left(\nabla \psi_{p}^{\dagger}\right)\left(\nabla \psi_{p}\right):-\frac{1}{6} \nabla^{2} \rho_{p}=\frac{4 \pi^{2}}{3}: \rho_{p}^{3}: .
$$

This expression may be familiar to those who work with the bosonization: it is the bosonic form of $H_{\mathrm{nl}}[1,11]$.

As a consequence of eq.(70) one derives:

$$
U H_{\mathrm{nl}} U^{\dagger}=\frac{4 \pi^{2} \tilde{v}_{\mathrm{F}}^{\prime}}{3} \sum_{p} \int d x U: \rho_{p}^{3}: U^{\dagger} .
$$

The expression under the integration sign is transformed as follows:

$$
\begin{array}{r}
U: \rho_{p}^{3}(x): U^{\dagger}= \\
\lim _{x^{\prime} \rightarrow x} U\left\{: \rho_{p}^{2}(x): \rho_{p}\left(x^{\prime}\right)-2 b_{p}\left(x-x^{\prime}\right) \rho_{p}(x)\right\} U^{\dagger} .
\end{array}
$$

Using our result for $U: \rho_{p}^{2}: U^{\dagger}$ we derive:

$$
\begin{array}{r}
U\left\{: \rho_{p}^{2}: \rho_{p}^{\prime}-2 b_{p}\left(x-x^{\prime}\right) \rho_{p}\right\} U^{\dagger}=(73) \\
{\left[:\left\{\rho_{p}^{u}+\rho_{-p}^{v}+\delta \rho_{p 0}\right\}^{2}:+c \Lambda^{2}\right]\left\{\left(\rho_{p}^{u}\right)^{\prime}+\left(\rho_{-p}^{v}\right)^{\prime}+\delta \rho_{p 0}\right\}} \\
-2 b_{p}\left(x-x^{\prime}\right)\left\{\rho_{p}^{u}+\rho_{-p}^{v}+\delta \rho_{p 0}\right\} .
\end{array}
$$

In this equality $\rho_{p}^{\prime}=\rho_{p}\left(x^{\prime}\right),\left(\rho_{p}^{u, v}\right)^{\prime}=\rho_{p}^{u, v}\left(x^{\prime}\right)$.

The cubic term in eq.(73) has to be normal ordered:

$$
\begin{array}{r}
:\left\{\rho_{p}^{u}+\rho_{-p}^{v}+\delta \rho_{p 0}\right\}^{2}:\left\{\left(\rho_{p}^{u}\right)^{\prime}+\left(\rho_{-p}^{v}\right)^{\prime}+\delta \rho_{p 0}\right\}= \\
:\left\{\rho_{p}^{u}+\rho_{-p}^{v}+\delta \rho_{p 0}\right\}^{2}\left\{\left(\rho_{p}^{u}\right)^{\prime}+\left(\rho_{-p}^{v}\right)^{\prime}+\delta \rho_{p 0}\right\}: \\
+2\left[\left(\hat{u} * b_{p} * \hat{u}\right)\left(x-x^{\prime}\right)+\left(\hat{v} * b_{-p} * \hat{v}\right)\left(x-x^{\prime}\right)\right] \times \\
\left\{\rho_{p}^{u}+\rho_{-p}^{v}+\delta \rho_{p 0}\right\} .
\end{array}
$$

Next one has to substitute eq.(74) into eq.(73) and collect similar terms. Using eq.(47) and definition eq.(51) it is easy to show now that:

$$
\begin{array}{r}
U: \rho_{p}^{3}: U^{\dagger}= \\
:\left\{\rho_{p}^{u}+\rho_{-p}^{v}+\delta \rho_{p 0}\right\}^{3}:+3 c \Lambda^{2}\left\{\rho_{p}^{u}+\rho_{-p}^{v}+\delta \rho_{p 0}\right\} .
\end{array}
$$

Therefore, up to terms $O\left(L^{0}\right)$ we find:

$$
U H_{\mathrm{nl}} U^{\dagger}=\frac{4 \pi^{2} \tilde{v}_{\mathrm{F}}^{\prime}}{3} \sum_{p} \int d x\left(:\left\{\rho_{p}^{u}+\rho_{-p}^{v}\right\}^{3}:+3 c \Lambda^{2} \rho_{p}\right)(.76)
$$

Now we need to expand the third order binomial:

$$
\begin{array}{r}
\sum_{p}:\left\{\rho_{p}^{u}+\rho_{-p}^{v}\right\}^{3}:=\sum_{p}\left[:\left(\rho_{p}^{u}\right)^{3}:+:\left(\rho_{p}^{v}\right)^{3}:\right. \\
\left.+3:\left(\rho_{p}^{u}\right)^{2}: \rho_{-p}^{v}+3:\left(\rho_{p}^{v}\right)^{2}: \rho_{-p}^{u}\right],
\end{array}
$$

and process the terms one by one. The first term:

$$
\begin{array}{r}
:\left(\rho_{p}^{u}\right)^{3}:=:\left(\rho_{p}+\rho_{p}^{w}\right)^{3}:= \\
: \rho_{p}^{3}:+3: \rho_{p}^{2} \rho_{p}^{w}:+3: \rho_{p}\left(\rho_{p}^{w}\right)^{2}:+:\left(\rho_{p}^{w}\right)^{3}: .
\end{array}
$$

We use the same trick which was exploited while obtaining eq.(54):

$$
\begin{array}{r}
\int d x:\left(\rho_{p}^{u}\right)^{3}:=\left(1+w_{0}\right)^{3} \int d x: \rho_{p}^{3}:+ \\
\int d x\left[\left\{3: \rho_{p}^{2} \rho_{p}^{w}:-3 w_{0}: \rho_{p}^{3}:\right\}+\right. \\
\left.\left\{3: \rho_{p}\left(\rho_{p}^{w}\right)^{2}:-3 w_{0}^{2}: \rho_{p}^{3}:\right\}+\left\{:\left(\rho_{p}^{w}\right)^{3}:-w_{0}^{3}: \rho_{p}^{3}:\right\}\right] .
\end{array}
$$

As with eq.(54) it is possible to convince ourselves that the expressions in curly brackets have higher scaling dimension than the scaling dimension of $: \rho_{p}^{3}:$. Indeed:

$$
\begin{array}{r}
: \rho_{p}^{2} \rho_{p}^{w}:-w_{0}: \rho_{p}^{3}:=\sum_{n=1}^{\infty} c_{n}^{w} \Lambda^{-2 n}: \rho_{p}^{2} \nabla^{2 n} \rho_{p}: \\
c_{n}^{w}=\frac{\Lambda^{2 n}}{(2 n) !} \int d x x^{2 n} w(x) .
\end{array}
$$

Dimension counting shows that the operators under the summation sign have the scaling dimensions of 5 and higher. This is bigger than 3 , the dimension of $: \rho_{p}^{3}$ :.

Acting in a similar fashion we establish for the second, third and fourth terms of eq.(77) that:

$$
\begin{array}{r}
\int d x:\left(\rho_{p}^{v}\right)^{3}:=v_{0}^{3} \int d x: \rho_{p}^{3}:+\int d x\left[:\left(\rho_{p}^{v}\right)^{3}:-v_{0}^{3}: \rho_{p}^{3}:\right],(82) \\
\int d x\left(:\left(\rho_{p}^{u}\right)^{2}: \rho_{-p}^{v}+:\left(\rho_{p}^{v}\right)^{2}: \rho_{-p}^{u}\right)=(83) \\
\left(u_{0}^{2} v_{0}+v_{0}^{2} u_{0}\right) \int d x: \rho_{p}^{2}: \rho_{-p}+ \\
\int d x\left(\left[:\left(\rho_{p}^{u}\right)^{2}: \rho_{-p}^{v}-u_{0}^{2} v_{0}: \rho_{p}^{2}: \rho_{-p}\right]+\right. \\
\left.\left[:\left(\rho_{p}^{v}\right)^{2}: \rho_{-p}^{u}-v_{0}^{2} u_{0}: \rho_{p}^{2}: \rho_{-p}\right]\right)
\end{array}
$$


where the expressions in square brackets has the scaling dimension 5 or higher.

Combining the above results we have:

$$
\begin{array}{r}
U H_{\mathrm{nl}} U^{\dagger}=\frac{4 \pi^{2} v_{\mathrm{F}, \mathrm{ex}}^{\prime}}{3} \int d x\left(: \rho_{\mathrm{L}}^{3}:+: \rho_{\mathrm{R}}^{3}:\right)+ \\
2 \pi g_{\mathrm{ex}}^{\prime} \int d x\left(: \rho_{\mathrm{L}}^{2}: \rho_{\mathrm{R}}+: \rho_{\mathrm{R}}^{2}: \rho_{\mathrm{L}}\right)+\frac{4 \pi^{2} \tilde{v}_{\mathrm{F}}^{\prime}}{3} h_{\mathrm{int}}^{(5)},
\end{array}
$$

$$
U H_{\mathrm{nl}} U^{\dagger}=\sum_{p} \int d x\left\{v_{\mathrm{F}, \mathrm{ex}}^{\prime}: \nabla \psi_{p}^{\dagger} \nabla \psi_{p}:+i g_{\mathrm{ex}}^{\prime} p\left(: \psi_{p}^{\dagger}\left(\nabla \psi_{p}\right):-:\left(\nabla \psi_{p}^{\dagger}\right) \psi_{p}:\right) \rho_{-p}\right\}+\frac{4 \pi^{2} \tilde{v}_{\mathrm{F}}^{\prime}}{3}\left(h_{\mathrm{int}}^{(5)}+3 c \Lambda^{2}\left(N_{\mathrm{L}}+N_{\mathrm{R}}\right)\right)
$$

Therefore, using the above formula and eq.(61) one finds the following expression for $H_{\mathrm{ex}}=U H_{0} U^{\dagger}$ :

$$
\begin{array}{r}
H_{\mathrm{ex}}=i v_{\mathrm{F}} \int d x\left(: \psi_{\mathrm{L}}^{\dagger} \nabla \psi_{\mathrm{L}}:-: \psi_{\mathrm{R}}^{\dagger} \nabla \psi_{\mathrm{R}}:\right)+\int d x d x^{\prime} \hat{g}\left(x-x^{\prime}\right) \rho_{\mathrm{L}}(x) \rho_{\mathrm{R}}\left(x^{\prime}\right)+ \\
v_{\mathrm{F}, \mathrm{ex}}^{\prime} \sum_{p} \int d x: \nabla \psi_{p}^{\dagger} \nabla \psi_{p}:+i g_{\mathrm{ex}}^{\prime} \sum_{p} p \int d x\left(: \psi_{p}^{\dagger}\left(\nabla \psi_{p}\right):-:\left(\nabla \psi_{p}^{\dagger}\right) \psi_{p}:\right) \rho_{-p}+\mu\left(N_{\mathrm{L}}+N_{\mathrm{R}}\right)+\pi \tilde{v}_{\mathrm{F}} h_{\mathrm{int}}^{(4)}+\frac{4 \pi^{2} \tilde{v}_{\mathrm{F}}^{\prime}}{3} h_{\mathrm{int}}^{(5)} .
\end{array}
$$

Since at $v_{\mathrm{F}}^{\prime} \neq 0$ the particle-hole symmetry is broken the bare value of the chemical potential is shifted: in the formula above $\mu=4 \pi^{2} c \tilde{v}_{\mathrm{F}}^{\prime} \Lambda^{2}$ whereas $\mu=0$ in Hamiltonian (8).

It is important to realize that $v_{\mathrm{F}, \mathrm{ex}}^{\prime}, g_{\mathrm{ex}}^{\prime}$ and $g$ in eq.(88) are not independent. By dividing eq.(85) on eq.(86) we obtain:

$$
\frac{v_{\mathrm{F}, \mathrm{ex}}^{\prime}}{g_{\mathrm{ex}}^{\prime}}=\frac{1}{2 \pi}\left(\frac{u_{0}^{2}+v_{0}^{2}}{u_{0} v_{0}}-1\right)
$$

Since

$$
\frac{u_{0}^{2}+v_{0}^{2}}{u_{0} v_{0}}=\frac{4 \pi v_{\mathrm{F}}}{g_{0}}
$$

we establish:

$$
v_{\mathrm{F}, \mathrm{ex}}^{\prime}=\left(\frac{2 v_{\mathrm{F}}}{g_{0}}-\frac{1}{2 \pi}\right) g_{\mathrm{ex}}^{\prime} .
$$

The deduced results can be summarized in the from of a theorem.

Theorem 1. Consider the Tomonaga-Luttinger model with nonlinear dispersion and the irrelevant operators $h_{\text {int }}^{(4,5)}$ whose scaling dimensions are 4 and higher. Such model is unitary equivalent to a system of free fermions with Hamiltonian (8) provided that parameters $v_{\mathrm{F}, \mathrm{ex}}^{\prime}, g_{\mathrm{ex}}^{\prime}$, $v_{\mathrm{F}}$ and $g_{0}$ satisfy eq.(91).

The theorem trivially implies that the TomonagaLuttinger model with Hamiltonian (88) whose parameters satisfy relation (91) is exactly soluble. This allows one to find the density-density propagator of eq.(88). Since the ground state $\left|0_{\mathrm{TL}}\right\rangle$ of Hamiltonian (88) is given by eq.(67) we derive:

$$
\begin{gathered}
\left\langle 0_{\mathrm{TL}}\left|\left\{\rho_{\mathrm{L} q}(\tau)+\rho_{\mathrm{R} q}(\tau)\right\}\left\{\rho_{\mathrm{L}-q}(0)+\rho_{\mathrm{R}-q}(0)\right\}\right| 0_{\mathrm{TL}}\right\rangle=(92) \\
\left\langle 0\left|U^{\dagger}\left\{\rho_{\mathrm{L} q}(\tau)+\rho_{\mathrm{R} q}(\tau)\right\} U U^{\dagger}\left\{\rho_{\mathrm{L}-q}(0)+\rho_{\mathrm{R}-q}(0)\right\} U\right| 0\right\rangle .
\end{gathered}
$$

Here, by the Green's function definition, $\rho_{p q}(\tau)=$ $\exp \left(\tau H_{\mathrm{ex}}\right) \rho_{p q} \exp \left(-\tau H_{\mathrm{ex}}\right)$, that is the evolution is controlled by $H_{\mathrm{ex}}$, eq. (88). The action of $U^{\dagger}=U^{-1}$ on the density and evolution operators is:

$$
U^{\dagger}\left(e^{\tau H_{\mathrm{ex}}} \rho_{p q} e^{-\tau H_{\mathrm{ex}}}\right) U=e^{\tau H_{0}}\left(u_{q} \rho_{p q}-v_{q} \rho_{-p q}\right) e^{-\tau H_{0}}(.93)
$$

Note the minus sign in the above transformation law. It differs from eq.(23) since here we apply $U^{\dagger}$ rather than $U$. Observe as well that the evolution of the density operators on the right-hand side of the above equation is set by the Hamiltonian (8). Thus:

$$
\begin{gathered}
\left\langle 0_{\mathrm{TL}}\left|\left\{\rho_{\mathrm{L} q}(\tau)+\rho_{\mathrm{R} q}(\tau)\right\}\left\{\rho_{\mathrm{L}-q}(0)+\rho_{\mathrm{R}-q}(0)\right\}\right| 0_{\mathrm{TL}}\right\rangle=(94) \\
\left(u_{q}-v_{q}\right)^{2}\left\langle 0\left|\left\{\rho_{\mathrm{L} q}+\rho_{\mathrm{R} q}\right\} e^{-\tau H_{0}}\left\{\rho_{\mathrm{L}-q}+\rho_{\mathrm{R}-q}\right\}\right| 0\right\rangle .
\end{gathered}
$$

The latter expectation value is easy to find. It is just a product of two free single-quasiparticle propagators corresponding to Hamiltonian (8). Therefore, Matsubara propagator for the Tomonaga-Luttinger model (88) is equal to:

$$
\mathcal{D}_{q}^{\mathrm{ex}}(\tau)=\left(u_{q}-v_{q}\right)^{2} \mathcal{D}_{q}^{0}(\tau),
$$

where $\mathcal{D}^{0}$ is the propagator for the Hamiltonian $H_{0}$ specified by eq.(13). At low $|q|$ this formula may be cast in a traditional for the bosonization literature way:

$$
\begin{array}{r}
\mathcal{D}_{q \omega}^{\mathrm{ex}}=\mathcal{K}_{q \omega}^{0}, \\
\mathcal{K}=\left(u_{0}-v_{0}\right)^{2}=\sqrt{\frac{2 \pi v_{\mathrm{F}}-g_{0}}{2 \pi v_{\mathrm{F}}+g_{0}}} .
\end{array}
$$


Here $\mathcal{K}$ is the usual Tomonaga-Luttinger liquid parameter.

The retarded propagator $D_{q \omega}^{\mathrm{ex}}$ and the spectral density $B_{q \omega}^{\mathrm{ex}}=-2 \operatorname{Im} D_{q \omega}^{\mathrm{ex}}$ are:

$$
\begin{array}{r}
D_{q \omega}^{\mathrm{ex}}=\frac{\mathcal{K}}{4 \pi \tilde{v}_{\mathrm{F}}^{\prime} q} \log \left\{\frac{\left(\tilde{v}_{\mathrm{F}} q-\tilde{v}_{\mathrm{F}}^{\prime} q^{2}\right)^{2}-(\omega+i 0)^{2}}{\left(\tilde{v}_{\mathrm{F}} q+\tilde{v}_{\mathrm{F}}^{\prime} q^{2}\right)^{2}-(\omega+i 0)^{2}}\right\}, \\
B_{q \omega}^{\mathrm{ex}}=\frac{\mathcal{K}}{2 \tilde{v}_{\mathrm{F}}^{\prime} q}\left\{\vartheta\left(\omega^{2}-\left(\tilde{v}_{\mathrm{F}} q-\tilde{v}_{\mathrm{F}}^{\prime} q^{2}\right)^{2}\right)-\right. \\
\left.\vartheta\left(\omega^{2}-\left(\tilde{v}_{\mathrm{F}} q+\tilde{v}_{\mathrm{F}}^{\prime} q^{2}\right)^{2}\right)\right\} \operatorname{sgn} \omega .
\end{array}
$$

Due to exact solubility the spectral density is zero everywhere outside the interval $\tilde{v}_{\mathrm{F}}|q|-\left|\tilde{v}_{\mathrm{F}}^{\prime}\right| q^{2}<|\omega|<$ $\tilde{v}_{\mathrm{F}}|q|+\left|\tilde{v}_{\mathrm{F}}^{\prime}\right| q^{2}$.

\section{NON-TECHNICAL OVERVIEW OF THE DERIVATIONS}

The presentation of the previous Section was rather technical. Below we will give a less formal account of the derivations performed so far.

It is obvious that the major source of technical complications are the zero modes and the irrelevant operators $h^{(4,5)}$. At the same time, they are the least important parts of the derived Hamiltonian (88). Thus, it is useful to redo the calculations of the Section II paying no attention to the zero modes and highly irrelevant operators. That way we will be able to capture easily the most important features of the algebraic manipulations without losing too much significant information.

Since the material presented in this Section is a refashioning of the discussion given above the reader should be prepared for some degree of redundancy.

Our technical goal is to start with the Hamiltonian of the free fermions with nonlinear dispersion $H_{0}$, eq.(8), and transform $H_{0}$ into $H_{\mathrm{ex}}$, eq.(88). We begin the execution of this program by writing $H_{0}$ as:

$$
H_{0}=\int d x \sum_{p} \pi \tilde{v}_{\mathrm{F}}: \rho_{p}^{2}(x):+\frac{4 \pi^{2} \tilde{v}_{\mathrm{F}}^{\prime}}{3}: \rho_{p}^{3}(x): .
$$

The validity of this equation can be checked with the help of eq.(43) and eq.(70). We already pointed out in the previous Section that the above equation may be thought of as the bosonic form of $H_{0}[1,11]$.

As in Sect.II we want to transform $H_{0}$ with the help of $U$ and find $U H_{0} U^{\dagger}$. Operator $U$ acts as a Bogoliubov rotation on the density operators, thus we may write (see eq.(35), eq.(50) and eq.(75)):

$$
\begin{array}{r}
U \rho_{p}(x) U^{\dagger}=u_{0} \rho_{p}(x)+v_{0} \rho_{-p}(x)+\ldots, \\
U: \rho_{p}^{2}(x): U^{\dagger}=:\left(u_{0} \rho_{p}(x)+v_{0} \rho_{-p}(x)\right)^{2}:+\ldots, \\
U: \rho_{p}^{3}(x): U^{\dagger}=:\left(u_{0} \rho_{p}(x)+v_{0} \rho_{-p}(x)\right)^{3}:+\ldots,
\end{array}
$$

where the ellipses stand for (a) additive constants, (b) zero modes terms $(c)$ irrelevant operators with high scaling dimensions and $(d)$ operators which are reduced to zero modes upon integration over $x$. As it was explained above such terms do nothing but cloud our view. Thus, we will neglect them.

Applying the rules eq.(101)-(103) to eq.(100) the following is derived:

$$
\begin{array}{r}
U H_{0} U^{\dagger}=\int d x \sum_{p} \pi \tilde{v}_{\mathrm{F}}:\left(u_{0} \rho_{p}+v_{0} \rho_{-p}\right)^{2}: \\
+\frac{4 \pi^{2} \tilde{v}_{\mathrm{F}}^{\prime}}{3}:\left(u_{0} \rho_{p}+v_{0} \rho_{-p}\right)^{3}:+\ldots \\
=\int d x \sum_{p} \pi \tilde{v}_{\mathrm{F}}\left(u_{0}^{2}+v_{0}^{2}\right): \rho_{p}^{2}:+\frac{4 \pi^{2} \tilde{v}_{\mathrm{F}}^{\prime}}{3}\left(u_{0}^{3}+v_{0}^{3}\right): \rho_{p}^{3}: \\
+2 \pi \tilde{v}_{\mathrm{F}} u_{0} v_{0} \rho_{p} \rho_{-p}+4 \pi^{2} \tilde{v}_{\mathrm{F}}^{\prime}\left(u_{0}^{2} v_{0}+v_{0}^{2} u_{0}\right): \rho_{p}^{2}: \rho_{-p}+\ldots,
\end{array}
$$

where the ellipsis stand for the zero modes and highly irrelevant operators. One notes that the term $: \rho_{p}^{2}$ : is proportional to $i p: \psi_{p}^{\dagger} \nabla \psi_{p}$ : and, thus, the coefficient in front of $: \rho_{p}^{2}$ : becomes $v_{\mathrm{F}}: v_{\mathrm{F}}=\tilde{v}_{\mathrm{F}}\left(u_{0}^{2}+v_{0}^{2}\right)$. Likewise, the coefficient of $\rho_{\mathrm{L}} \rho_{\mathrm{R}}$ becomes $g_{0}$ (see eq.(60)), the coefficient in front of $: \rho_{p}^{2}: \rho_{-p}$ is $g_{\mathrm{ex}}^{\prime}$ (see eq.(86)) and the coefficient of $: \rho_{p}^{3}$ : is $v_{\mathrm{F}, \mathrm{ex}}^{\prime}$ (see eq.(85)). Consequently, we obtain:

$$
\begin{gathered}
H_{\mathrm{ex}}=U H_{0} U^{\dagger}=(105) \\
\int d x \sum_{p} i p v_{\mathrm{F}}: \psi_{p}^{\dagger} \nabla \psi_{p}:+v_{\mathrm{F}, \mathrm{ex}}^{\prime}: \nabla \psi_{p}^{\dagger} \nabla \psi_{p}:+\frac{1}{2} g_{0} \rho_{p} \rho_{-p} \\
+i p g_{\mathrm{ex}}^{\prime}\left(: \psi_{p}^{\dagger}\left(\nabla \psi_{p}\right):-:\left(\nabla \psi_{p}^{\dagger}\right) \psi_{p}:\right) \rho_{-p}+\ldots
\end{gathered}
$$

The previous equation coincide with eq.(88) up to zero modes and highly irrelevant operators. The coefficients $g_{\mathrm{ex}}^{\prime}$ and $v_{\mathrm{F}}^{\prime}$ satisfy eq.(91). This concludes our nontechnical overview of the exactly soluble Hamiltonian derivation. Transcending many technical details discussed in Section II we discover that the method of calculating $H_{\mathrm{ex}}$ is in fact fairly simple.

Another question we want to discuss here is the meaning of the exact solubility line eq.(91). Consider the generic Hamiltonian $H_{\mathrm{G}}$ :

$$
H_{\mathrm{G}}=H_{\mathrm{TL}}+H_{\mathrm{nl}}+H_{\mathrm{int}}^{\prime},
$$

whose parameters do not satisfy any specific relation. It is possible to find the transformation $V$ of the form eq.(14) which diagonalizes the first term of the above equation. For the detailed calculations one should consult Ref.[9] or our derivation of eq.(61). The heuristic explanation, however, is easy. Consider the 'bosonic' form of $H_{\mathrm{TL}}$ :

$$
H_{\mathrm{TL}}=\frac{\pi v_{\mathrm{F}}}{L} \sum_{p q}\left(\rho_{p q} \rho_{p-q}+\frac{g_{q}}{2 \pi v_{\mathrm{F}}} \rho_{p q} \rho_{-p-q}\right)+\ldots
$$

Application of $V$ produces the following:

$$
\begin{array}{r}
V H_{\mathrm{TL}} V^{\dagger}= \\
\frac{\pi v_{\mathrm{F}}}{L} \sum_{p q}\left[u_{q}^{2}+v_{q}^{2}+\frac{g_{q}}{\pi v_{\mathrm{F}}} u_{q} v_{q}\right] \rho_{p q} \rho_{p-q}+ \\
{\left[\frac{g_{q}}{2 \pi v_{\mathrm{F}}}\left(u_{q}^{2}+v_{q}^{2}\right)+2 u_{q} v_{q}\right] \rho_{p q} \rho_{-p-q}+\ldots}
\end{array}
$$


By adjusting $u_{q}$ and $v_{q}$ we can kill the interaction term $\rho_{p} \rho_{-p}$. For this to happens $u, v$ must satisfy the equation:

$$
\frac{g_{q}}{2 \pi v_{\mathrm{F}}}\left(u_{q}^{2}+v_{q}^{2}\right)+2 u_{q} v_{q}=0
$$

or, equivalently:

$$
\text { th } 2 \alpha_{q}=-\frac{g_{q}}{2 \pi v_{\mathrm{F}}} .
$$

If this condition is met we have:

$$
V H_{\mathrm{TL}} V^{\dagger}=i \tilde{v}_{\mathrm{F}} \int d x\left(: \psi_{\mathrm{L}}^{\dagger} \nabla \psi_{\mathrm{L}}:-: \psi_{\mathrm{R}}^{\dagger} \nabla \psi_{\mathrm{R}}:\right)+\ldots
$$

where the product $\rho_{p} \rho_{p}$ was expressed in terms of the fermionic operators $\psi, \psi^{\dagger}$ with the help of eq.(43).

There exists another transformation $V^{\prime}$ of the general form (14) but with a different set of coefficients $\alpha^{\prime}$ which diagonalizes the sum $\left(H_{\mathrm{nl}}+H_{\mathrm{int}}^{\prime}\right)$ :

$$
V^{\prime}\left(H_{\mathrm{nl}}+H_{\mathrm{int}}^{\prime}\right)\left(V^{\prime}\right)^{\dagger}=\tilde{v}_{\mathrm{F}}^{\prime} \int d x \sum_{p}: \nabla \psi_{p}^{\dagger} \nabla \psi_{p}:+\ldots
$$

For this to take place the parameters $\alpha^{\prime}$ must satisfy the relation:

$$
2 \pi v_{\mathrm{F}}^{\prime}+g^{\prime}\left(1+2 \mathrm{cth} 2 \alpha_{0}^{\prime}\right)=0
$$

where $\alpha_{0}^{\prime}$ equals to $\alpha_{q}^{\prime}$ at $q=0$. The derivations of the two above equations is similar to the proof of equations (110) and (111). The idea of these derivations is to write $V^{\prime}\left(H_{\mathrm{nl}}+H_{\mathrm{int}}^{\prime}\right)\left(V^{\prime}\right)^{\dagger}$ in terms of $\rho$ 's:

$$
\begin{array}{r}
V^{\prime}\left(H_{\mathrm{nl}}+H_{\mathrm{int}}^{\prime}\right)\left(V^{\prime}\right)^{\dagger}=V^{\prime}\left[\int d x \frac{4 \pi^{2} v_{\mathrm{F}}^{\prime}}{3}\left(: \rho_{\mathrm{L}}^{3}:+: \rho_{\mathrm{R}}^{3}:\right)(114)\right. \\
\left.+2 \pi g^{\prime}\left(: \rho_{\mathrm{L}}^{2}: \rho_{\mathrm{R}}+: \rho_{\mathrm{R}}^{2}: \rho_{\mathrm{L}}\right)+\ldots\right]\left(V^{\prime}\right)^{\dagger},
\end{array}
$$

and adjust $\alpha_{0}^{\prime}$ of $V^{\prime}$ in such a way as to remove $: \rho_{p}^{2}: \rho_{-p}$ terms. This requirement lead us to eq.(113). Once the latter terms are removed what remains is proportional to $: \rho_{\mathrm{L}}^{3}:+: \rho_{\mathrm{R}}^{3}:$. This expression is proportional to $H_{\mathrm{nl}}$ and, therefore, is diagonal.

If the values of $v_{\mathrm{F}}, v_{\mathrm{F}}^{\prime}, g$ and $g^{\prime}$ are arbitrary then $V \neq V^{\prime}$. Consequently, the whole Hamiltonian $H_{\mathrm{G}}$ cannot be diagonalized with the help of the canonical transformation of the form eq.(14). However, if we demand that $V=V^{\prime}$ or, equivalently, $\alpha=\alpha^{\prime}$ such diagonalization becomes possible and the system becomes soluble. One can check that the exact solubility condition (91) may be derived from the equation $\alpha_{0}=\alpha_{0}^{\prime}$. Thus, one can say that the exact solubility condition guarantees the simultaneous diagonalization of the whole Hamiltonian.

\section{GENERIC TOMONAGA-LUTTINGER MODEL WITH NONLINEAR DISPERSION}

In this Section we will discuss how the above findings can be applied to a generic model of the one-dimensional spinless fermions.
The most general Hamiltonian is:

$$
H_{\mathrm{G}}=H_{\mathrm{TL}}+H_{\mathrm{nl}}+H_{\mathrm{int}}^{\prime}+H^{(4)},
$$

where $H^{(4)}$ stands for operators whose scaling dimensions are 4 and higher. Here no special relation between $v_{\mathrm{F}}^{\prime}$ and $g^{\prime}$ is assumed. Neither do we suppose that $H^{(4)}$ is equal to the linear combination of $h^{(4)}$ and $h^{(5)}$ from eq. (88). To avoid clutter we do not show the chemical potential term explicitly for it can be accounted trivially.

It is convenient to write Hamiltonian $H_{\mathrm{G}}$ in the form:

$$
\begin{array}{r}
H_{\mathrm{G}}=H_{\mathrm{ex}}+\delta H_{\mathrm{nl}}+\delta H^{(4)}, \\
\delta H_{\mathrm{nl}}=\delta v_{\mathrm{F}}^{\prime} \int d x \sum_{p}: \nabla \psi_{p}^{\dagger} \nabla \psi_{p}: \\
\delta v_{\mathrm{F}}^{\prime}=v_{\mathrm{F}}^{\prime}-v_{\mathrm{F}, \mathrm{ex}}^{\prime} .
\end{array}
$$

The term $\delta H^{(4)}$ contains those operators whose scaling dimension are 4 and higher and which are not absorbed into $H_{\mathrm{ex}}$. The constant $\delta v_{\mathrm{F}}^{\prime}$ and $\delta H^{(4)}$ measure the deviation of the Hamiltonian $H_{\mathrm{G}}$ from the exact solubility line. We want to apply the transformation $V$ of the form eq.(14) to $H_{\mathrm{G}}$ such that the transformed Hamiltonian contains no marginal interaction operator $H_{\text {int }}$. The remaining irrelevant operators can be treated perturbatively.

We already know how the first two terms of eq.(116) are transformed under the action of $V$. The unknown entity is $V \delta H^{(4)} V^{\dagger}$. To discuss the action of $V$ on $\delta H^{(4)}$ it is useful to introduce the notation:

$$
\begin{array}{r}
h_{\text {kin }}=i \int d x\left(: \psi_{\mathrm{L}}^{\dagger} \nabla \psi_{\mathrm{L}}:-: \psi_{\mathrm{R}}^{\dagger} \nabla \psi_{\mathrm{R}}:\right), \\
h_{\mathrm{int}}=\int d x \rho_{\mathrm{L}} \rho_{\mathrm{R}}, \\
h_{\mathrm{int}}^{\prime}=\int d x \sum_{p} i p \rho_{-p}\left(: \psi_{p}^{\dagger}\left(\nabla \psi_{p}\right):-:\left(\nabla \psi_{p}^{\dagger}\right) \psi_{p}:\right), \\
h_{\mathrm{nl}}=\int d x \sum_{p}: \nabla \psi_{p}^{\dagger} \nabla \psi_{p}:
\end{array}
$$

These operators exhaust the list of operators whose scaling dimension is lower than 4 and we can write the most general expression:

$$
\begin{array}{r}
V \delta H^{(4)} V^{\dagger}= \\
\beta_{\text {kin }} h_{\text {kin }}+\beta_{\text {int }} h_{\text {int }}+\beta_{\text {nl }} h_{\mathrm{nl}}+\beta_{\text {int }}^{\prime} h_{\text {int }}^{\prime}+\delta \bar{H}^{(4)} .
\end{array}
$$

The coefficients $\beta$ 's are functions of $\alpha$ 's which specify $V$.

Now we must consider two cases: (i) when $\beta_{\text {int }}=0$ and (ii) when $\beta_{\text {int }} \neq 0$.

Let us start with (i). The transformation $V$ with

$$
\alpha_{0}=-\frac{1}{2} \operatorname{th}^{-1}\left(\frac{g_{0}}{2 \pi v_{\mathrm{F}}}\right)
$$

acts on $H_{\mathrm{G}}$ as follows:

$$
V H_{\mathrm{G}} V^{\dagger}=H_{0}+\left(\delta \tilde{v}_{\mathrm{F}}^{\prime} h_{\mathrm{nl}}+\delta \tilde{g}^{\prime} h_{\mathrm{int}}^{\prime}\right)+V \delta H^{(4)} V^{\dagger}
$$




$$
\begin{array}{r}
=\left[H_{0}+\beta_{\text {kin }} h_{\text {kin }}+\left(\delta \tilde{v}_{\mathrm{F}}^{\prime}+\beta_{\mathrm{nl}}\right) h_{\mathrm{nl}}\right] \\
+\left(\delta \tilde{g}^{\prime}+\beta_{\mathrm{int}}^{\prime}\right) h_{\mathrm{int}}^{\prime}+\delta \bar{H}^{(4)}, \\
\delta \tilde{v}_{\mathrm{F}}^{\prime}=\left(u_{0}^{3}-v_{0}^{3}\right) \delta v_{\mathrm{F}}^{\prime}, \\
\delta \tilde{g}^{\prime}=2 \pi\left(v_{0}^{2} u_{0}-u_{0}^{2} v_{0}\right) \delta v_{\mathrm{F}}^{\prime} .
\end{array}
$$$$
\delta \tilde{v}_{\mathrm{F}}^{\prime}=\left(u_{0}^{3}-v_{0}^{3}\right) \delta v_{\mathrm{F}}^{\prime},(126)
$$

In the first line of eq.(125) the expression in the round brackets corresponds to $V \delta v_{\mathrm{F}}^{\prime} h_{\mathrm{nl}} V^{\dagger}$.

Eq.(125) can be reformulated in a more compact way:

$$
\begin{array}{r}
V H_{\mathrm{G}} V^{\dagger}=\bar{H}_{0}+\delta H_{\mathrm{irr}}, \\
\bar{H}_{0}=H_{0}+\beta_{\mathrm{kin}} h_{\mathrm{kin}}+\left(\delta \tilde{v}_{\mathrm{F}}^{\prime}+\beta_{\mathrm{nl}}\right) h_{\mathrm{nl}}, \\
\delta H_{\mathrm{irr}}=\left(\delta \tilde{g}^{\prime}+\beta_{\mathrm{int}}^{\prime}\right) h_{\mathrm{int}}^{\prime}+\delta \bar{H}^{(4)} .
\end{array}
$$

Hamiltonian $\bar{H}_{0}$ is quadratic in the fermionic field operators. It is similar to $H_{0}$ but has renormalized parameter values. Likewise, $\delta \bar{H}^{(4)}$ is the renormalized version of $\delta H^{(4)}$.

We see that deviation from the exact solubility line eq.(91) in $\delta v_{\mathrm{F}}^{\prime}$ has two effects: (a) renormalization of $\tilde{v}_{\mathrm{F}}$ and $\tilde{v}_{\mathrm{F}}^{\prime}$ and $(b)$ introduction of the interactions between the quasiparticles (operator $\delta H_{\text {irr }}$ ). Fortunately, these interactions are irrelevant in the renormalization group sense. Thus, their influence could be accounted perturbatively. For that reason let us introduce the notation:

$$
H_{\mathrm{qp}}=V H_{\mathrm{G}} V^{\dagger} \text {, }
$$

which suggests that the transformed operator $V H_{\mathrm{G}} V^{\dagger}$ represents the Hamiltonian of the weakly interacting quasiparticles.

Next we study the case (ii). This situation is not much more difficult than (i). From the field-theoretical point of view $\beta_{\text {int }} \neq 0$ means that irrelevant operators contribute to the renormalization of the marginal operator coupling constant $g$. The correct way to address the problem is to fix $\alpha_{0}$ by a generalized version of eq.(124). The value of $\alpha_{0}$ must be determined by the requirement that the quasiparticle Hamiltonian

$$
\begin{array}{r}
H_{\mathrm{qp}}=\gamma_{\mathrm{kin}} h_{\mathrm{kin}}+\gamma_{\mathrm{int}} h_{\mathrm{int}}+\gamma_{\mathrm{nl}} h_{\mathrm{nl}}+ \\
\gamma_{\mathrm{int}}^{\prime} h_{\mathrm{int}}^{\prime} \\
+\delta \bar{H}^{(4)}
\end{array}
$$

$\left(\gamma\right.$ 's are functions of $\left.\alpha_{0}\right)$ contains no $h_{\text {int }}$ :

$$
\gamma_{\text {int }}\left(\alpha_{0}\right)=0 \text {. }
$$

It is easy to demonstrate that eq.(133) is equivalent to eq.(124) if $\beta_{\text {int }}=0$.

Once $\alpha_{0}$ is determined according to eq.(133) the expression (132) attains the form of eq.(128). Thus, we prove

Theorem 2. A generic Tomonaga-Luttinger model with nonlinear dispersion is unitary equivalent to a system of the free quasiparticles with irrelevant interactions.

\section{CALCULATIONS OF OBSERVABLES}

What does Theorem 2 imply for evaluation of the physical properties of $H_{\mathrm{G}}$ ? The irrelevance of corrections $\delta H_{\text {irr }}$ means that the ground state properties of Hamiltonian $H_{\mathrm{G}}$ can be approximated by the ground state properties of $\bar{H}_{0}$. Should we be interested in corrections those could be found perturbatively.

Further, for an observable $\mathcal{O}(x)$ the correlation function

$$
\begin{array}{r}
\mathcal{G}_{\mathcal{O}}=-\left\langle 0_{\mathrm{G}}\left|\mathcal{T}_{\tau}\left\{\mathcal{O}(x, \tau) \mathcal{O}^{\dagger}(0,0)\right\}\right| 0_{\mathrm{G}}\right\rangle, \\
\mathcal{O}(x, \tau)=\mathrm{e}^{\tau H_{\mathrm{G}}} \mathcal{O}(x) \mathrm{e}^{-\tau H_{\mathrm{G}}}, \\
\left|0_{\mathrm{G}}\right\rangle-H_{\mathrm{G}} \text { ground state }
\end{array}
$$

may be expressed as:

$$
\begin{array}{r}
\mathcal{G}_{\mathcal{O}}=-\left\langle 0_{\mathrm{qp}}\left|\mathcal{T}_{\tau}\left\{\tilde{\mathcal{O}}(x, \tau) \tilde{\mathcal{O}}^{\dagger}(0,0)\right\}\right| 0_{\mathrm{qp}}\right\rangle, \\
\tilde{\mathcal{O}}(x, \tau)=\mathrm{e}^{\tau H_{\mathrm{qp}}}\left(V \mathcal{O}(x) V^{\dagger}\right) \mathrm{e}^{-\tau H_{\mathrm{qp}}} \\
\left|0_{\mathrm{qp}}\right\rangle-\text { ground state of } H_{\mathrm{qp}}
\end{array}
$$

If the observable $\mathcal{O}$ is such that $V \mathcal{O} V^{\dagger}$ has a very complicated form the practical evaluation of eq.(137) may be difficult to carry out. An example of such situation is $\mathcal{O} \equiv \psi_{p}$. At present the author does not know how the single-fermion propagator for the case of nonzero $v_{\mathrm{F}}^{\prime}$ can be calculated.

On the other hand, if the object $V \mathcal{O} V^{\dagger}$ has some simple representation in terms of the quasiparticle degrees of freedom one can evaluate $\mathcal{G}_{\mathcal{O}}$ with the help of the usual perturbative expansion in orders of $\delta H_{\text {irr }}$. For example, consider calculation of the density-density propagator: $\mathcal{O} \equiv\left(\rho_{\mathrm{L}}+\rho_{\mathrm{R}}\right)$. In that instance $V \mathcal{O} V^{\dagger}$ is a linear combination of the density operators. The zeroth order expression for such propagator is:

$$
\mathcal{D}_{q \omega}=\mathcal{D}_{q \omega}^{\mathrm{ex}}+O\left(\left(\delta H_{\mathrm{irr}}\right)^{2}\right) .
$$

The corrections in orders of $\delta H_{\text {irr }}$ could be determined with the help of field-theoretical techniques.

Note, that the actual calculation of $O\left(\left(\delta H_{\text {irr }}\right)^{2}\right)$ corrections may be quite nontrivial. Indeed, numerical evidence [14] and heuristic analytical arguments [15] indicates that $D_{q \omega}$ of the generic model diverges stronger than $D_{q \omega}^{\mathrm{ex}}$. This suggests that the perturbation theory for $D_{q \omega}$ requires a reliable resummation technique.

However, we are not always interested in the propagator itself. In fact, there are situations when a few low-order diagrams contributing to $D_{q \omega}$ would suffice. For example, consider some quantity $r$ proportional to an integral over $D_{q \omega}$. [An important example of such quantity is the Coulomb drag resistivity [6]: $r \propto$ $\int d q d \omega f(q, \omega)\left(\operatorname{Im} D_{q \omega}\right)^{2}$, where $f$ is some temperaturedependent kernel.] Unlike the propagator itself, $r$ remains finite. This is so because the divergences of $D_{q \omega}$ due to $O\left(\left(\delta H_{\text {irr }}\right)^{2}\right)$ corrections are very weak. Thus, the integration of $D_{q \omega}$ can be successfully performed with the result:

$$
r=r^{\mathrm{ex}}+\delta r,
$$

where quasiparticle interaction correction $|\delta r|<\infty$ may be non-analytic. 
Regarding the quasiparticle interaction corrections due to $\delta H_{\text {irr }}$ we would like to make a remark. Operator $\left(\delta \tilde{g}^{\prime}+\beta_{\text {int }}^{\prime}\right) h_{\text {int }}^{\prime}$ is the least irrelevant part of $\delta H_{\text {irr }}$. Consequently, we expect that at small momenta the corrections due to $\left(\delta \tilde{g}^{\prime}+\beta_{\text {int }}^{\prime}\right) h_{\text {int }}^{\prime}$ are stronger than those due to $\delta \bar{H}^{(4)}$. Therefore, the most important contribution to the low momenta corrections are $\propto\left(\delta \tilde{g}^{\prime}+\beta_{\text {int }}^{\prime}\right)^{2}$. Thus, there is a hypersurface in the generic one-dimensional model parameter space where the low momenta corrections drastically weakens. This hypersurface is fixed by the equation

$$
\delta \tilde{g}^{\prime}+\beta_{\mathrm{int}}^{\prime}=0 .
$$

We can interpret this as a manifestation of the exact solubility line eq.(91).

As a practical application one can calculate the hypersurface (142) for the spinless Hubbard-like model

$$
H_{\mathrm{hub}}=\sum_{i, j} t(i-j) c_{i}^{\dagger} c_{j}+\mu \sum_{i} n_{i}+u \sum_{i} n_{i} n_{i+1}
$$

in the limit of small interaction $u \ll t$. Using the decomposition:

$$
c_{i}=\psi_{\mathrm{L}}\left(x_{i}\right) e^{-i k_{\mathrm{F}} x_{i}}+\psi_{\mathrm{R}}\left(x_{i}\right) e^{i k_{\mathrm{F}} x_{i}},
$$

one can calculate $v_{\mathrm{F}}, v_{\mathrm{F}}^{\prime}, g$ and $g^{\prime}$ in terms of $t(i)$ and $u$. As soon as this task is done the equations for those quantities must be substituted into eq.(91) and the limit $u \rightarrow 0$ is taken. This gives:

$$
v_{\mathrm{F}, \mathrm{ex}}^{\prime}=a v_{\mathrm{F}} \operatorname{ctg}\left(k_{\mathrm{F}} a\right),
$$

where $a$ is the lattice constant and the values of $v_{\mathrm{F}}$ and $v_{\mathrm{F}, \mathrm{ex}}^{\prime}$ are functionals of $t(i)$.

What are the phenomenological manifestations of eq.(145)? As explained above, on the surface fixed by the latter equation and in the limit $u \ll t$ the scattering of the quasiparticles is drastically reduced. As a consequence, the propagator $D_{q \omega}$ calculated for the Hamiltonian satisfying eq.(145) differs qualitatively from the propagator of the generic Hamiltonian eq.(143). For example, the divergence of the propagator due to quasiparticle scattering might be weaker. Such prediction may be tested numerically.

\section{COMPARISON WITH OTHER WORK}

The issue of nonzero $v_{\mathrm{F}}^{\prime}$ has a long history. Traditionally, it has been approached within the framework of the bosonization $[1,11,13]$. The disadvantage of the bosonization is that the perturbative expansion in orders of $v_{\mathrm{F}}^{\prime}$ is highly singular.

To circumvent this latter difficulty the authors of Ref.[12] used the random phase approximation to find $D_{q \omega}$. Their spectral function differs qualitatively from eq.(99). We believe that such a result is an artefact of the random phase approximation.
Alternatively, in several recent papers $[6,7]$ the use of the 'fermionic' methods was advocated. The idea behind them is that away from the mass surface the perturbation theory in $g$ is permissible without bosonization. That is, the authors chose to work with the original fermions but at a price: the perturbation theory results cannot be applied straightforwardly near the mass surface.

The proposed method was used to calculate the highfrequency tail of the propagator. The latter technique is complimentary to the exact solution: it is easily implemented at high frequency but it is problematic at the mass surface where the exact solution works.

In Ref. [15] the heuristic argument was put forward suggesting that the spectral function $B$ of $H_{\mathrm{G}}$ diverges at some line of $(q, \omega)$ plane. This finding is corroborated qualitatively by the numerical work [14]. How does this compare against calculations presented here?

Obviously, our spectral function $B^{\text {ex }}$ of the exactly soluble model is finite. This does not contradict to Ref.[14, 15]: the propagator of the generic model $H_{\mathrm{G}}$ is affected by the quasiparticle interaction which induces the extra divergence. Thus, the propagator calculation requires an adequate resummation technique, as we pointed out in the previous Section. We may hypothesize that the argumentation of Ref. [15] may be directly applied to the quasiparticle Hamiltonian $H_{\mathrm{qp}}$. Such 'marriage' of the two approaches possesses a clear advantage: both marginal and irrelevant interactions are accounted for to all orders. Further speculations are to be postponed until thorough investigation.

To conclude, we have shown that the TomonagaLuttinger model with specific parameter values is equivalent to the free fermions. At this point the TomonagaLuttinger Hamiltonian is exactly soluble. Away from the exact solubility the model may be mapped on a system of the fermions with irrelevant interactions. Although, under such circumstances the model is not soluble exactly, yet, it can be investigated with the help of the perturbation theory.

\section{ACKNOWLEDGEMENTS}

The author is grateful for the support provided by the Dynasty Foundation, by RFBR through grant 03-0216626 and by Russian federal program "Leading scientific schools" NSh-1694.2003.2.

\section{APPENDIX}

In this Appendix we prove certain relations required by the presentation in the main text.

First, we derive eq.(39). Our initial step is to prove:

$$
\begin{array}{r}
: \rho_{p}(x) \rho_{p}(y):=: \psi_{p}^{\dagger}(x) \psi_{p}(x) \psi_{p}^{\dagger}(y) \psi_{p}(y):+ \\
s_{p}(x-y): \psi_{p}(x) \psi_{p}^{\dagger}(y):+s_{p}(x-y): \psi_{p}^{\dagger}(x) \psi_{p}(y):
\end{array}
$$


The proof goes as follows:

$$
\begin{array}{r}
\rho_{p}(x) \rho_{p}(y)=: \psi_{p}^{\dagger}(x) \psi_{p}(x):: \psi_{p}^{\dagger}(y) \psi_{p}(y):= \\
s_{p}^{2}(x-y)+: \psi_{p}^{\dagger}(x) \psi_{p}(x) \psi_{p}^{\dagger}(y) \psi_{p}(y):+ \\
s_{p}(x-y): \psi_{p}(x) \psi_{p}^{\dagger}(y):+s_{p}(x-y): \psi_{p}^{\dagger}(x) \psi_{p}(y):,
\end{array}
$$

where we used eq. (10). Taking into consideration eq.(41) and $b_{p}=s_{p}^{2}$ one obtains the desired formula eq. (146).

Once eq.(146) is established eq.(39) follows. We send $y \rightarrow x$ and note that $: \psi_{p}^{\dagger}(x) \psi_{p}(x) \psi_{p}^{\dagger}(y) \psi_{p}(y):=0$ if $x=$ $y$. Indeed:

$$
\begin{aligned}
& : \psi_{p}^{\dagger}(x) \psi_{p}(x) \psi_{p}^{\dagger}(y) \psi_{p}(y):= \\
& -: \psi_{p}^{\dagger}(y) \psi_{p}(x) \psi_{p}^{\dagger}(x) \psi_{p}(y):
\end{aligned}
$$

Since the quartic term vanishes we need to evaluate the quadratic terms of eq.(146) only. To calculate them at $x=y$ one can utilize the L'Hospital's rule:

$$
\begin{array}{r}
\lim _{y \rightarrow x}\left[s_{p}(x-y)\left\{: \psi_{p}(x) \psi_{p}^{\dagger}(y):+: \psi_{p}^{\dagger}(x) \psi_{p}(y):\right\}\right]=( \\
\frac{i p}{2 \pi}\left\{: \psi_{p}(x) \nabla \psi_{p}^{\dagger}(x):+: \psi_{p}^{\dagger}(x) \nabla \psi_{p}(x):\right\}
\end{array}
$$

where we took into account that

$$
\frac{\partial}{\partial y}\left(\frac{1}{s_{p}(x-y)}\right)=\frac{2 \pi}{i p} .
$$

It is obvious now that eq.(39) is valid.

Next, we want to prove eq.(70). The procedure is similar to the one we have used just now. It is possible to show:

$$
\begin{aligned}
& : \rho_{p}(x) \rho_{p}(y) \rho_{p}(z):=: \psi_{p}^{\dagger}(x) \psi_{p}(x) \psi_{p}^{\dagger}(y) \psi_{p}(y) \psi_{p}^{\dagger}(z) \psi_{p}(z): \\
& +s_{p}(x-y)\left\{: \psi_{p}^{\dagger}(x) \psi_{p}(y) \psi_{p}^{\dagger}(z) \psi_{p}(z):-: \psi_{p}^{\dagger}(y) \psi_{p}(x) \psi_{p}^{\dagger}(z) \psi_{p}(z):\right\} \\
& +s_{p}(x-z)\left\{: \psi_{p}^{\dagger}(x) \psi_{p}(z) \psi_{p}^{\dagger}(y) \psi_{p}(y):-: \psi_{p}^{\dagger}(z) \psi_{p}(x) \psi_{p}^{\dagger}(y) \psi_{p}(y):\right\} \\
& +s_{p}(y-z)\left\{: \psi_{p}^{\dagger}(y) \psi_{p}(z) \psi_{p}^{\dagger}(x) \psi_{p}(x):-: \psi_{p}^{\dagger}(z) \psi_{p}(y) \psi_{p}^{\dagger}(x) \psi_{p}(x):\right\} \\
& +s_{p}(y-z) s_{p}(x-z)\left\{: \psi_{p}(x) \psi_{p}^{\dagger}(y):-: \psi_{p}^{\dagger}(x) \psi_{p}(y):\right\}+s_{p}(x-y) s_{p}(x-z)\left\{: \psi_{p}(y) \psi_{p}^{\dagger}(z):-: \psi_{p}^{\dagger}(y) \psi_{p}(z):\right\} \\
& -s_{p}(x-y) s_{p}(y-z)\left\{: \psi_{p}(x) \psi_{p}^{\dagger}(z):-: \psi_{p}^{\dagger}(x) \psi_{p}(z):\right\} \text {. }
\end{aligned}
$$

Like eq.(146), this equality was deduced by writing $\rho \rho \rho$ as a product of six fermionic fields which is then expressed as a sum of the normal ordered expressions. If we put in the above equation $x=y=z$ only terms quadratic in the fermionic fields would survive. This is a consequence of the fermionic field anticommutativity (see eq.(148)). Since we are interested in $x=y=z$ limit we have to handle the quadratic part of eq.(151) only.

Let us define an operator-valued function:

$$
\begin{gathered}
G(x, y, z)= \\
s_{p}(y-z) s_{p}(x-z)\left\{: \psi_{p}(x) \psi_{p}^{\dagger}(y):-: \psi_{p}^{\dagger}(x) \psi_{p}(y):\right\} \\
+s_{p}(x-y) s_{p}(x-z)\left\{: \psi_{p}(y) \psi_{p}^{\dagger}(z):-: \psi_{p}^{\dagger}(y) \psi_{p}(z):\right\} \\
-s_{p}(x-y) s_{p}(y-z)\left\{: \psi_{p}(x) \psi_{p}^{\dagger}(z):-: \psi_{p}^{\dagger}(x) \psi_{p}(z):\right\} .
\end{gathered}
$$

It coincides with the quadratic part of eq.(151). Therefore, $: \rho_{p}^{3}(x):=G(x, x, x)$. We will demonstrate that

$$
\begin{array}{r}
G(x, x, x)=: \rho_{p}^{3}(x):= \\
\frac{3}{4 \pi^{2}}\left(:\left(\nabla \psi_{p}^{\dagger}(x)\right)\left(\nabla \psi_{p}(x)\right):-\frac{1}{6} \nabla^{2} \rho_{p}(x)\right) .
\end{array}
$$

We begin the proof by an observation:

$$
\begin{aligned}
s_{p}(x-y) s_{p}(y-z)= & s_{p}(x-y) s_{p}(x-z)+ \\
& s_{p}(y-z) s_{p}(x-z) .
\end{aligned}
$$

The formula can be checked directly with the help of eq.(11). We transform eq.(152):

$$
\begin{gathered}
G(x, y, z)=(155) \\
s_{p}(x-y) s_{p}(x-z)\left\{:\left[\psi_{p}^{\dagger}(x)-\psi_{p}^{\dagger}(y)\right] \psi_{p}(z):+ \text { h.c. }\right\}- \\
s_{p}(y-z) s_{p}(x-z)\left\{:\left[\psi_{p}^{\dagger}(y)-\psi_{p}^{\dagger}(z)\right] \psi_{p}(x):+ \text { h.c. }\right\} .
\end{gathered}
$$

It is convenient to define another operator-valued function $F$ :

$$
\begin{array}{r}
G(x, y, z)=s_{p}(x-z)[F(x, y, z)-F(y, z, x)], \\
F(x, y, z)= \\
s_{p}(x-y)\left\{:\left[\psi_{p}^{\dagger}(x)-\psi_{p}^{\dagger}(y)\right] \psi_{p}(z):+ \text { h.c. }\right\} .
\end{array}
$$

Function is well-defined for any values of $x, y$ and $z$.

It is important to note that $F$ is invariant under the transposition of its two first arguments:

$$
\begin{array}{r}
F(x, y, z)=\left(\delta(x-y)-s_{p}(y-x)\right) \times \\
\left\{-:\left[\psi_{p}^{\dagger}(y)-\psi_{p}^{\dagger}(x)\right] \psi_{p}(z):+ \text { h.c. }\right\}=F(y, x, z),
\end{array}
$$

since $\delta(x-y):\left[\psi_{p}^{\dagger}(x)-\psi_{p}^{\dagger}(y)\right] \psi_{p}(z):=0$. Consequently, we can rewrite the expression for $G$ :

$$
G(x, y, z)=s_{p}(x-z)[F(x, y, z)-F(z, y, x)] .
$$


The expression in the square brackets vanishes at $x=z$. Thus, function $G$ is well-defined everywhere. Sending $z \rightarrow x$ we obtain with the help of the L'Hospital's rule:

$$
G(x, x, x)=\left.\frac{i p}{2 \pi} \frac{\partial}{\partial z}[F(x, x, z)-F(z, x, x)]\right|_{z=x}
$$

Applying the L'Hospital's rule the following equality is derived:

$$
F(x, x, z)=\frac{p}{2 \pi i}\left\{:\left(\nabla \psi_{p}^{\dagger}(x)\right) \psi_{p}(z):+ \text { h.c }\right\}
$$

Consequently:

$$
\left.\frac{\partial}{\partial z} F(x, x, z)\right|_{z=x}=\frac{p}{\pi i}:\left(\nabla \psi_{p}^{\dagger}(x)\right)\left(\nabla \psi_{p}(x)\right):
$$

We calculate:

$$
\begin{gathered}
\left.\frac{\partial}{\partial z} F(z, x, x)\right|_{z=x}=\frac{p}{4 \pi i}\left\{:\left(\nabla^{2} \psi_{p}^{\dagger}(x)\right) \psi_{p}(x):+ \text { h.c. }\right\}(163) \\
=\frac{p}{4 \pi i}\left\{\nabla^{2} \rho_{p}(x)-2:\left(\nabla \psi_{p}^{\dagger}(x)\right)\left(\nabla \psi_{p}(x)\right):\right\},
\end{gathered}
$$

by applying the L'Hospital's rule yet again.

Combining the results for $G$ and derivatives of $F$ we prove eq.(153) which, in turn, proves eq.(70).
[1] F.D.M. Haldane, J. Phys. C: Solid State Phys. 14, 2585 (1981).

[2] A.O. Gogolin, A.A. Nersesyan, A.M. Tsvelik, Bosonization and Strongly Correlated Systems, (Cambridge University Press, Cambridge, UK, 1998).

[3] X. G. Wen, Phys. Rev. B 41, 12838 (1990).

[4] Stefan Kehrein, Phys. Rev. Lett. 83, 4914 (1999).

[5] Tai-Kai Ng, Phys. Rev. B 68, 041101(R) (2003).

[6] M. Pustilnik, E.G. Mishchenko, L.I. Glazman, and A.V. Andreev, Phys. Rev. Lett. 91, 126805 (2003).

[7] Sofian Teber, Eur. Phys. J. B 52, 233 (2006), and condmat/0511257.

[8] A.V. Rozhkov, Phys. Rev. B 68, 115108 (2003).

[9] A.V. Rozhkov, Eur. Phys. J. B, 47, 193 (2005).

[10] Jan von Delft, Herbert Schoeller, Ann. Phys. (Leipzig)
7, 225 (1998), and cond-mat/9805275

[11] Peter Kopietz, "Bosonization of Interacting Fermions in Arbitrary Dimensions", (Springer, Heidelberg, 1997).

[12] Peyman Pirooznia and Peter Kopietz, condmat/0512494.

[13] K.V. Samokhin, J. Phys. Condens. Matter 10 L533 (1998).

[14] R.G. Pereira, J. Sirker, J.-S. Caux, R. Hagemans, J.M. Maillet, S.R. White, and I. Affleck, Phys. Rev. Lett., 96, 257202 (2006).

[15] M. Pustilnik, M. Khodas, A. Kamenev, and L.I. Glazman, Phys. Rev. Lett. 96, 196405 (2006) and condmat/0603458. 